\title{
Superior Mesenteric Vein Thrombosis as a complication following Jejunal lymphoma resection: A Case Report
}

\author{
D. N. Goyal ${ }^{1}$, Venkateswara Rao Katta ${ }^{1}$, Vijay Kumar V. R. N. ${ }^{1}$, \\ Sumeera Farhath ${ }^{1}$, Ashok Kumar V. ${ }^{1}$, Bhaskara Narayana K. ${ }^{2}$ \\ ${ }^{I}$ Department of General Surgery, MNR Medical College and Hospital, Sangareddy, Andhra Pradesh, India \\ ${ }^{2}$ Department of Radiology, MNR Medical College and Hospital, Sangareddy, Andhra Pradesh, India
}

\begin{abstract}
We report a 65 year old female who presented with Left iliac fossa mass for three months with pain for ten days. Ultrasound and CT scans have revealed a lymphoma involving the descending colon. Colonoscopy was normal. At mini laparotomy, the mass was found to be arising from jejunum, with impending obstruction. A Jejunal resection was carried out. Following stable first three post-operative days, the patient developed septicemia. Review CT scan revealed, non-perfused, gangrenous bowel. At relook laparotomy, there was extensive small bowel gangrene along the drainage territories of Superior mesenteric vein. Patient succumbed to septic shock on fourth post-operative day.
\end{abstract}

Key Words: Jejunal Lymphoma, Superior Mesenteric Vein (SMV) Thrombosis, Gangrene small bowel, Computerized Tomography (CT). Mesenteric Vein Thrombosis (MVT)

\section{Introduction}

MVT was first recognized as cause of intestinal infarction by Elliott in 1895 [1]. It was not until 1935, that this condition was differentiated from mesenteric arterial occlusion by Warren and Eberhard[2]. Different pathophysiologic mechanisms have been postulated for acute thrombus formation because of local factors (e.g. recent splenectomy or pancreatitis), as distinct from those associated with systemic hypercoagulable states (e.g. protein C deficiency or MPD) [3, 4]. Thus, local factors are associated with initial thrombus formation in the large veins whereas hypercoagulability leads to thrombosis beginning in the intramural venules, vasa recta and venous arcades [5]

\section{Case Report}

We report a 65 year old female with left iliac fossa mass for three months. She developed a dull aching pain over the past ten days over the swelling. There were no obstructive or urinary symptoms. There was no history of weight loss or bleeding per rectum. There was also no history of malaise or tiredness nor there chills and rigors. Her past history was noncontributory and she is not taking any medications. She was anemic at presentation and there were no palpable lymph nodes. The mass was measuring $8 \mathrm{cms}$ on palpation. It was fixed to deeper structures and not mobile.

An ultrasound and CT scans have confirmed the mass to be arising from sigmoid colon with necrotic mass with in the mesentery. Colonoscopy revealed normal mucosa. After optimization of general condition, we performed a mini laparotomy, which then had to be converted to a laparotomy as the mass was seen to be arising from jejunum with dilatation of Jejunal loops proximal to the mass, with impending obstruction, as seen in Fig.1. A Jejunal resection was carried out. Standard thromboprohylaxis with sub cutaneous low molecular weight heparin was given in the post-operative period.

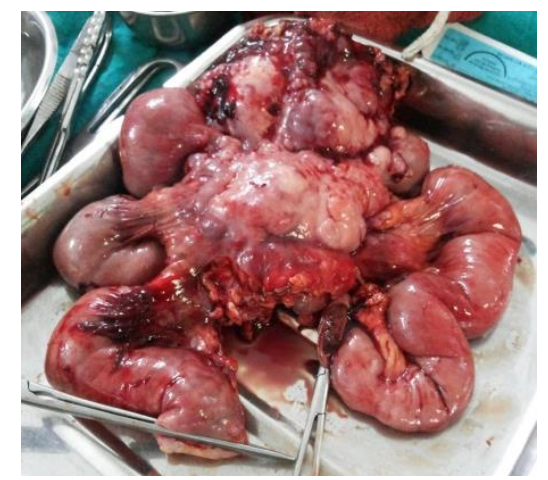

Figure 1: Resected specimen of Jejunum with mesentery showing impending obstruction. 
After stable first three days, patient developed signs of septicemia, at which stage a CT scan was done, which revealed non perfused small bowel with intra mural gas and anastomotic leak. At relook laparotomy, there was extensive small bowel gangrene from jejunum up to terminal ileum. The distal $30 \mathrm{cms}$ of terminal ileum was spared. The superior mesenteric artery was patent throughout its length. The superior mesenteric vein was patent at its confluence with splenic vein but distally, there was no flow. An intra-operative diagnosis of Superior mesenteric vein thrombosis with anastomotic leak was made. After a bolus dose of intra venous heparin, extensive small bowel resection was carried out as shown in Fig.2, with anastomosis between terminal ileum and distal duodenum. The patient had persistent septcemic shock and passed away the day after. We suspect combination of hypercoagulable state post major abdominal surgery and tumour embolization as possible factors for superior mesenteric vein thrombosis. The final histopathology of resected specimen was of Non-Hodgkin's lymphoma of jejunum.

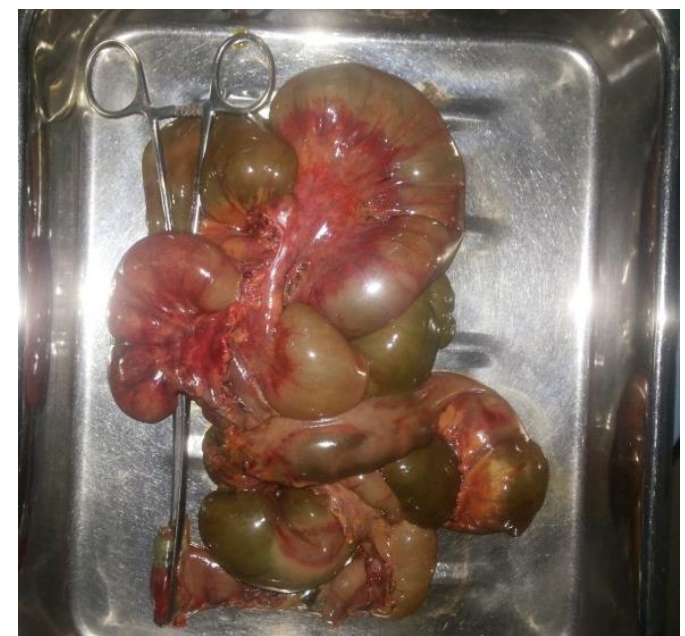

Figure 2: extensive gangrenous small bowel which was resected.

\section{Discussion}

In 1952, McCune[6] found that immediate infarction was associated with the presence of thrombi within the arcuate veins, vasa recta and intramural vessels, often without involvement of major venous trunks. Acute MVT begins either in the smaller peripheral vessels and extends proximally in patients with systemic thrombophilia, or in the larger splanchnic trunks and spreads distally in patients with local intra-abdominal causes of hypercoagulability. Although acute MVT is responsible for only about 3-15\% of cases of AMI, a high index of suspicion is needed for accurate diagnosis [5]. Evidence of infarction mandates emergent surgical consultation and abdominal exploration and/or aggressive intravascular therapy in order to save as much intestine as possible. Short-term mortality largely depends on whether infarction is present and has improved over time because of earlier diagnosis and aggressive therapy [5].

\section{References:}

[1] Elliot JW. The operative relief of gangrene of intestine due to occlusion of the mesenteric vessels. Ann Surg 1895; 21: 9-23.

[2] Warren S, Eberhard TP. Mesenteric venous thrombosis. Surg Gynecol Obstet 1935; 61: 102-121.

[3] Brandt LJ, Feuerstadt P. Intestinal ischemia. In: Feldman M, Friedman LS, Brandt LJ (eds). Sleisenger and Fordtran's Gastrointestinal and Liver Disease: Pathophysiology/Diagnosis/Management. 9th edition. Philadelphia: WB Saunders, 2010.

[4] Kumar S, Sarr M, Kamath PS. Mesenteric venous thrombosis. N Engl J Med 2001; 345: 1683-1688.

[5] Ian G Harnik, Lawrence J Brandt. Mesenteric venous thrombosis. Vasc Med 2010 15: 407.

[6] McCune WS, Keshishian JM, Frankhauser RL. Mesenteric thrombosis following blunt abdominal trauma. Ann Surg 1952; 135: 606-614. 